\title{
A shallow-flow model for the propagation of tsunamis over complex geometries and mobile beds
}

\author{
D. A. S. Conde ${ }^{1}$, M. A. V. Baptista ${ }^{2}$, C. Sousa Oliveira ${ }^{3}$, and R. M. L. Ferreira ${ }^{1}$ \\ ${ }^{1}$ CEHIDRO, Instituto Superior Técnico, TU Lisbon, Lisbon, Portugal \\ ${ }^{2}$ Centro de Geofísica da Universidade de Lisboa, IDL and Instituto Superior de Engenharia de Lisboa, Lisbon, Portugal \\ ${ }^{3}$ ICIST, Instituto Superior Técnico, TU Lisbon, Lisbon, Portugal
}

Correspondence to: D. A. S. Conde (daniel.conde@ist.utl.pt)

Received: 11 December 2012 - Published in Nat. Hazards Earth Syst. Sci. Discuss.: Revised: 17 May 2013 - Accepted: 31 May 2013 - Published: 16 October 2013

\begin{abstract}
A distinguishable feature of overland tsunami propagation is the incorporation of solids within the flow column, either sediment from the natural environment or remains from built infrastructure. This article describes a 2DH (two-dimensional horizontal) mathematical model particularly suited for tsunami propagation over complex and dynamic geometries, such as river and estuarine mobile beds. The discretization scheme is based on a finite-volume method using a flux-splitting technique featuring a reviewed Roe-Riemann solver, with appropriate source-term formulations to ensure full conservativeness. The model is validated with laboratory data and paleo-tsunami evidence. As a forecasting application, it is applied to a tsunami scenario in the Tagus estuary, an effort justified by the numerous catastrophic tsunamis that are known to have struck this location over the past two millennia. The obtained results show that, despite the significant differences in Lisbon's layout and morphology, a 1755-like tsunami would still inflict a devastating impact on this major city.
\end{abstract}

\section{Introduction}

Tsunamis are mostly generated by the vertical displacement of the water column due to underwater earthquakes, subaerial or underwater landslides, among other less frequent causes. They are translatory waves that propagate over oceanic basins with low amplitude, invisible to the naked eye. Approaching the continental slope, the shoaling effect slows down the wave, leading to higher wave heights and revealing the incoming tsunami. As it heads towards the shoreline, the waves may break and form bores, a stage where the tsunami peaks in destructive capacity. The imparted momentum will force debris and natural sediment to become incorporated into the propagating wavefront, causing its mechanical properties to become those of a granular-fluid flow. This kind of interaction, during both inland wave propagation and drawdown stages, is expected to considerably reshape the existing bed morphology, as seen in the 2004 and 2011 events in the Indian Ocean and Japan, respectively.

The Tagus estuary in Portugal is particularly prone to devastating tsunamis as shown by Baptista and Miranda (2009) in a recent revision of the tsunami catalog in Portugal for the past two millennia. Such exposure motivates detailed modelling studies that aim to assess possible inundation extents for the current topographic conditions of the region. Part of the aforementioned modelling work has been recently developed (Baptista et al., 2011), although clearance for both conceptual and numerical improvements does exist.

The objective of this paper is to present a $2 \mathrm{DH}$ (twodimensional horizontal) mathematical model suitable to simulate the propagation of discontinuous flows, such as a tsunami propagating inland. The core of the model is a hyperbolic system of partial differential equations expressing mass and momentum conservation principles for both water and granular material. Sediment transport is modelled with a non-equilibrium model, featuring an imbalance between actual and capacity sediment transport (Ferreira et al., 2009). The finite-volume discretization scheme, firstly proposed by Murillo and Garcia-Navarro (2010), is based on a fully conservative flux-splitting technique featuring a reviewed Roe-Riemann solver, including sediment concentration terms (Canelas et al., 2013). 
This work is divided into six sections. An overview of the conceptual model is provided in Sect. 2 while Sect. 3 presents the most relevant features of the employed numerical approach. The validation tests, emphasizing bed interaction capabilities and historical evidence comparisons are shown in Sect. 4. The application of this model, herein designated STAV-2D, to a well-documented tsunami scenario in the Tagus estuary dating back to AD 1755, is presented in Sect. 5. The paper is completed with a set of conclusions in Sect. 6.

\section{Conceptual model}

\subsection{Overview}

The conceptual model herein presented is based on the shallow-water equations featuring dynamic bed geometries and sediment transport modelled via the difference between capacity bedload discharge and local solid discharge. This conceptual model is closed in terms of flow resistance, capacity bedload discharge and a specific return-to-capacity parameter by a modified closure model (described in the following section) originally proposed by Ferreira et al. (2009), based on the granular flow paradigm (Chapman and Cowling, 1970; Jenkins and Richman, 1988) merged with the continuum hypothesis.

The discretization technique provides fully conservative solutions to initial value problems (IVPs). Nonhomogeneous terms related to the bed slope in momentum conservation laws are discretized consistently with steady solutions, such as water resting in hydrostatic equilibrium. This is a relevant property since it supports the concept of wellbalanced schemes (Toro, 2001; Vazquez-Cendon, 1999). The model further features a consistent wetting-drying algorithm, entropy conditions to ensure the physical feasibility of shocks and a revised Courant condition (Murillo and GarciaNavarro, 2010). The discretization method also allows bodyfitting unstructured grids. These can optimize the computational workload by using large cells, where flow gradients are expected to be mild, and small cells in specified areas, such as buildings or other obstacles, where gradients are usually quite large.

\subsection{Conservation equations}

The conservation equations for total mass, momentum in both horizontal directions, and sediment mass transported with the flow and deposited on the bed are given by

$$
\begin{aligned}
& \partial_{\mathrm{t}} h+\partial_{x}(h u)+\partial_{y}(h v)=-\partial_{\mathrm{t}} Z_{\mathrm{b}} \\
& \partial_{\mathrm{t}}(u h)+\partial_{x}\left(u^{2} h+\frac{1}{2} g h^{2}\right)+\partial_{y}(u v h) \\
& \quad=-g h \partial_{x} Z_{\mathrm{b}}-\frac{1}{\rho_{\mathrm{m}}} \partial_{x} h T_{x x}-\frac{1}{\rho_{\mathrm{m}}} \partial_{y} h T_{x y}-\frac{\tau_{\mathrm{b}, x}}{\rho_{\mathrm{m}}}
\end{aligned}
$$

$$
\begin{gathered}
\partial_{\mathrm{t}}(v h)+\partial_{x}(v u h)+\partial_{y}\left(v^{2} h+\frac{1}{2} g h^{2}\right) \\
-g h \partial_{y} Z_{\mathrm{b}}-\frac{1}{\rho_{\mathrm{m}}} \partial_{x} h T_{y x}-\frac{1}{\rho_{\mathrm{m}}} \partial_{y} h T_{y y}-\frac{\tau_{\mathrm{b}, y}}{\rho_{\mathrm{m}}} \\
\partial_{t}\left(C_{\mathrm{m}} h\right)+\partial_{x}\left(C_{\mathrm{m}} h u\right)+\partial_{y}\left(C_{\mathrm{m}} h v\right)=-(1-p) \partial_{t} Z_{\mathrm{b}}
\end{gathered}
$$

and

$\frac{\partial Z_{\mathrm{b}}}{\partial t}=\frac{q_{\mathrm{s}}-q_{\mathrm{s}}^{*}}{\Lambda}(1-p)^{-1}$,

where $x$ and $y$ are the 2-D horizontal space coordinates; $t$ is time; $h$ is the fluid height above bed elevation; $Z_{\mathrm{b}}, u$ and $v$ are the depth-averaged velocities in the $x$ and $y$ directions, respectively; $\partial_{t} Z_{\mathrm{b}}$ is the net vertical mass flux between the bed and the flow; $\rho_{\mathrm{m}}$ and $C_{\mathrm{m}}$ represent the depth-averaged density and concentration of the mixture, respectively; $\tau_{\mathrm{b}, x_{i}}$ represents the bottom slope momentum sources, with $x_{i}$ as the $x$ or $y$ direction; $T_{i j}$ are the depth-averaged turbulent stresses; $q_{\mathrm{S}}$ is the solid discharge; $q_{\mathrm{s}}^{*}$ is its capacity value; and their difference, mediated by an adaptation length $\Lambda$, renders a time-integrated net mass flux, expressed as volume per unit area. The inertia of the granular material and the added pressure are neglected in the momentum conservation Eqs. (2) and (3). A full derivation of these equations can be found in Canelas (2010) or Conde (2012).

\subsection{Closure equations}

The bed shear stress, $\tau_{\mathrm{b}}$, is given by

$\tau_{\mathrm{b}}=C_{\mathrm{f}}|\boldsymbol{u}|^{2} \rho_{\mathrm{m}}$,

where $\rho_{\mathrm{m}}$ is the average density of the flow. The friction coefficient, $C_{\mathrm{f}}$, can be provided by the Manning-Strickler law or, for debris flows, by a formula derived by Ferreira et al. (2009). The turbulent stress tensor, $T_{i j}$, is such that

$T_{i j}=\rho_{\mathrm{w}} v_{T}\left(\frac{\partial u_{i}}{\partial x_{j}}+\frac{\partial u_{j}}{\partial x_{i}}\right)$

where $\rho_{\mathrm{w}}$ is the water density and $\nu_{T}$ is the eddy viscosity, expressed as a simplified zero-equation $\kappa-\epsilon$ model (Pope, 2000).

Several bedload formulas can be used in STAV-2D to describe capacity bedload transport, among them the wellknown Meyer-Peter and Muller (1948) and Bagnold (1966) formulas. A specific formula for stratified flows (sheet flow) and debris flows is also incorporated (Ferreira et al., 2009). The bedload discharge is defined as

$q_{\mathrm{s}}^{*}=C_{c}^{*}\left|\boldsymbol{u}_{c}\right|^{2} h_{c}$,

where $C_{c}^{*}$ and $\boldsymbol{u}_{c}$ are the layer-averaged capacity concentration and velocity in the contact load layer, respectively. Assuming that the thickness of this layer, $h_{c}$, is related to the 
flux of kinetic energy associated with the fluctuating motion of transported grains (Ferreira et al., 2009), one has

$$
\frac{h_{c}}{d_{\mathrm{s}}}=m_{1}+m_{2} \theta
$$

where $d_{\mathrm{S}}$ is the characteristic particle diameter, and $m_{1}$ and $m_{2}$ are parameters that depend on the mechanical properties, diameter and density of the sediment particles and on the fluid viscosity, as theoretically derived and calibrated by Ferreira et al. (2009). The Shields parameter is given by $\theta=C_{\mathrm{f}}\left|\boldsymbol{u}_{c}\right|^{2} /\left(g d_{\mathrm{s}}(s-1)\right)$ and $s=\rho_{\mathrm{s}} / \rho_{\mathrm{W}}$ is the particles specific gravity, where $\rho_{\mathrm{S}}$ is the granular material density. The bedload layer velocity is taken as in Ferreira et al. (2006),

$\boldsymbol{u}_{c}=\boldsymbol{u}\left(\frac{h_{c}}{h}\right)^{1 / 6}$.

The capacity concentration, $C_{c}^{*}$, was also derived by Ferreira et al. (2009), assuming equilibrium of the frictional sublayer. One obtains

$C_{c}^{*}=\frac{\theta}{\tan \left(\varphi_{\mathrm{b}}\right)\left(m_{1}+m_{2} \theta\right)}$,

where $\tan \left(\varphi_{\mathrm{b}}\right)$ is the dynamic friction angle of the granular material - the ratio between normal and shear granular stresses (Bagnold, 1966). The adaptation length can be chosen by the user. In this work, a formula proposed by Canelas et al. (2013) was employed.

\section{Numerical model}

\subsection{Discretization technique}

The hyperbolic non-homogeneous system, composed by quasi-linear first order Eqs. (1)-(4), can be rewritten in a more compact notation as

$\partial_{t} \boldsymbol{U}(\boldsymbol{V})+\partial_{x} \boldsymbol{F}(\boldsymbol{U})+\partial_{y} \boldsymbol{G}(\boldsymbol{U})=\boldsymbol{H}(\boldsymbol{U})$

with

$$
\begin{gathered}
\boldsymbol{V}=\left[\begin{array}{c}
h \\
u \\
v \\
C_{\mathrm{m}}
\end{array}\right] ; \quad \boldsymbol{U}=\left[\begin{array}{c}
h \\
u h \\
v h \\
C_{\mathrm{m}} h
\end{array}\right] ; \quad \boldsymbol{F}=\left[\begin{array}{c}
u h \\
u^{2} h+\frac{1}{2} g h^{2} \\
u v h \\
C_{\mathrm{m}} h u
\end{array}\right] ; \\
\boldsymbol{G}=\left[\begin{array}{c}
v h \\
v u h \\
v^{2} h+\frac{1}{2} g h^{2} \\
C_{\mathrm{m}} h v
\end{array}\right] ; \quad \boldsymbol{H}=\left[\begin{array}{c}
-\partial_{t} Z_{\mathrm{b}} \\
-g h \partial_{x} Z_{\mathrm{b}}-\tau_{x} / \rho \\
-g h \partial_{y} Z_{\mathrm{b}}-\tau_{y} / \rho \\
-\partial_{t} Z_{\mathrm{b}}(1-p)
\end{array}\right] ;
\end{gathered}
$$

where $\boldsymbol{V}$ and $\boldsymbol{U}$ are the vectors of primitive and conservative dependant variables, respectively; $\boldsymbol{F}$ and $\boldsymbol{G}$ are the fluxes in the $x$ and $y$ direction, respectively; $\boldsymbol{H}$ is the source term
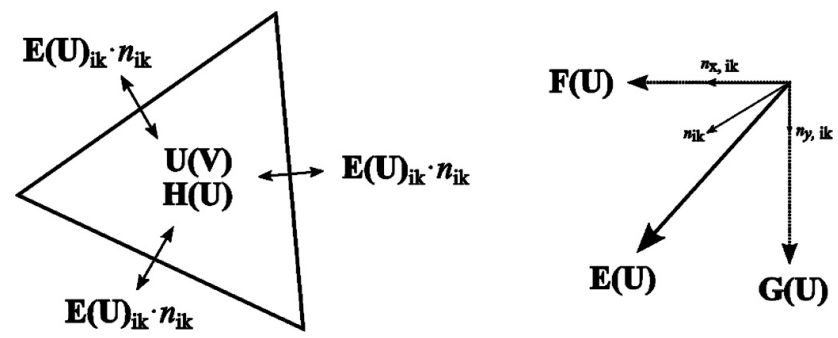

Fig. 1. Scheme of variables involved in the numerical model.

vector; and $\tau_{i}=\tau_{\mathrm{b}, i}+\partial_{x_{i}} h T_{i i}+\partial_{x_{j}} h T_{\mathrm{ij}}$. By employing Godunov's finite-volume based discretization technique (LeVeque, 2002), the system (Eq. 11) is integrated within each cell $i$. Figure 1 summarizes the concept behind the numerical scheme, where dependant variables inside each cell, $\boldsymbol{V}(\boldsymbol{U})$ and $\boldsymbol{H}(\boldsymbol{U})$, are updated according to the fluxes on each cell edge, $\boldsymbol{E}(\boldsymbol{U})=\boldsymbol{F}(\boldsymbol{U}) \cdot \boldsymbol{n}_{x}+\boldsymbol{G}(\boldsymbol{U}) \cdot n_{y}$.

This yields the following explicit flux-based finite-volume method:

$\boldsymbol{U}_{i}^{n+1}=\boldsymbol{U}_{i}^{n}+\Delta t\left(\boldsymbol{R}_{i}^{n+1}\right)$

$-\frac{\Delta t}{A_{i}} \sum_{k=1}^{3} L_{k} \sum_{m=1}^{4}\left(\tilde{\lambda}^{(m)} \alpha^{(m)}-\beta^{(m)}\right)_{i k}^{-} \tilde{\boldsymbol{e}}_{i k}^{(m)}$

where $\Delta t$ is the time step, obeying a Courant (CFL) condition (LeVeque, 2002); $A_{i}$ is the cell area; $L_{k}$ is the edge length; $\tilde{\boldsymbol{e}}^{(m)}$ is the $m$-th approximate eigenvector, given by,

$$
\begin{gathered}
\tilde{\boldsymbol{e}}_{i k}^{(1)}=\left[\begin{array}{c}
1 \\
\tilde{u}-\tilde{c} n_{x} \\
\tilde{v}-\tilde{c} n_{y} \\
\tilde{C_{m}}
\end{array}\right]_{i k}, \quad \tilde{\boldsymbol{e}}_{i k}^{(2)}=\left[\begin{array}{c}
0 \\
-\tilde{c} n_{y} \\
\tilde{c} n_{x} \\
0
\end{array}\right]_{i k}, \\
\tilde{\boldsymbol{e}}_{i k}^{(3)}=\left[\begin{array}{c}
1 \\
\tilde{u}+\tilde{c} n_{x} \\
\tilde{v}+\tilde{c} n_{y} \\
\tilde{C_{m}}
\end{array}\right]_{i k}, \quad \tilde{\boldsymbol{e}}_{i k}^{(4)}=\left[\begin{array}{c}
0 \\
0 \\
0 \\
1
\end{array}\right]_{i k} ;
\end{gathered}
$$

and $\tilde{\lambda}^{(m)}$ is the $m$-th approximate eigenvalue defined as

$$
\begin{aligned}
& \tilde{\lambda}_{i k}^{(1)}=(\tilde{\boldsymbol{u}} \cdot \boldsymbol{n}-\tilde{c})_{i k}, \quad \tilde{\lambda}_{i k}^{(2)}=(\tilde{\boldsymbol{u}} \cdot \boldsymbol{n})_{i k}, \\
& \tilde{\lambda}_{i k}^{(3)}=(\tilde{\boldsymbol{u}} \cdot \boldsymbol{n}+\tilde{c})_{i k}, \quad \tilde{\lambda}_{i k}^{(4)}=(\tilde{\boldsymbol{u}} \cdot \boldsymbol{n})_{i k} .
\end{aligned}
$$

The approximate velocities in the $x$ and $y$ directions, $\tilde{u}$ and $\widetilde{v}$, respectively, approximate free-surface disturbances celerity, $\widetilde{c}$, and approximate concentration, $\widetilde{C}_{m}$, and are defined as

$$
\begin{gathered}
\tilde{u}_{i k}=\frac{u_{i} \sqrt{h_{i}}+u_{j} \sqrt{h_{j}}}{\sqrt{h_{i}}+\sqrt{h_{j}}} ; \quad \tilde{v}_{i k}=\frac{v_{i} \sqrt{h_{i}}+v_{j} \sqrt{h_{j}}}{\sqrt{h_{i}}+\sqrt{h_{j}}} ; \\
\tilde{c}_{i k}=\sqrt{g \frac{h_{i}+h_{j}}{2}} ; \quad \tilde{C}_{m_{i k}}=\frac{C_{m_{i}} \sqrt{h_{i}}+C_{m_{j}} \sqrt{h_{j}}}{\sqrt{h_{i}}+\sqrt{h_{j}}} .
\end{gathered}
$$


The wave strengths associated with conservative fluxes are denoted by $\alpha^{(m)}$, whereas $\beta^{(m)}$ are the wave strengths associated with non-conservative fluxes, $g h \partial_{x_{i}} Z_{\mathrm{b}}$. The vector $\boldsymbol{R}_{i}^{n+1}=\left(\left[\partial_{t} Z_{\mathrm{b}}-\tau_{x} / \rho_{\mathrm{m}}-\tau_{y} / \rho_{\mathrm{m}} \partial_{t} Z_{\mathrm{b}}(1-p)\right]^{T}\right)_{i}^{n+1}$ is the parcel of the source terms not compatible with a non-conservative flux treatment. Terms of the form $g h \partial_{x_{i}} Z_{\mathrm{b}}$ are not physical fluxes, but they are treated as such to obtain a well-balanced scheme (Murillo and Garcia-Navarro, 2010). Only the negative part of the eigenvalues, $\tilde{\lambda}_{i k}^{(m)}$, and $\beta_{i k}^{(m)}$ coefficients are considered, ensuring that conserved variables are updated only with incoming fluxes. The approximate eigenvectors, approximate eigenvalues and both types of wave strengths are computed as proposed by Murillo and Garcia-Navarro (2010). Further details on the numerical scheme can be found in Canelas (2010), namely what concerns wetting-drying algorithms, entropy conditions, Courant condition (CFL) and discretization of turbulent stresses.

\section{Model validation}

\subsection{Stoker solution for dam-break flows over fixed flat smooth beds}

Setups that admit theoretical solutions comprise an efficient method for validating numerical results. In this test the dambreak problem over fixed flat smooth beds, an IVP that can be theoretically solved (Stoker, 1957) was employed. The nondimensional parameters describing the initial conditions are given by

$\alpha \equiv\left(h_{\mathrm{R}}+\left|\min \left(0, Z_{\mathrm{b}_{1}}\right)\right|\right) /\left(h_{\mathrm{L}}+\max \left(0, Z_{\mathrm{b}_{1}}\right)\right)$

$\delta \equiv Z_{\mathrm{b}_{1}} /\left(h_{\mathrm{L}}+\max \left(0, Z_{\mathrm{b}_{1}}\right)\right)$

where $h_{\mathrm{R}}$ and $h_{\mathrm{L}}$ are the initial fluid depths on the right and left states of the discontinuity, respectively, and $Z_{\mathrm{b}_{1}}$ is the bed elevation on the left side (Fig. 2), provided that the right side coincides with the reference horizontal plane (Ferreira et al., 2009). According to the Lax theorem, the solution exists under the form of two waves separated by a constant state (LeVeque, 2002).

With $h_{\mathrm{L}}=10 \mathrm{~m}, \alpha=0.0$ and $\delta=0.0$, the solution presents an expansion wave and a wetting advancing front where the intermediate constant state and the right shock are collapsed. The discretization is $2 \mathrm{DH}$ with triangular cells, whose average edge is $0.65 \mathrm{~m}$, in a channel $10.0 \mathrm{~m}$ wide. Figure 3a shows the numerical results obtained with $\mathrm{CFL}=0.8$, along with the theoretical solution. This test showed, in general, a good agreement between STAV-2D results and the exact solution. However, there are a few minor issues with the numerical results, the most noticeable of which being a

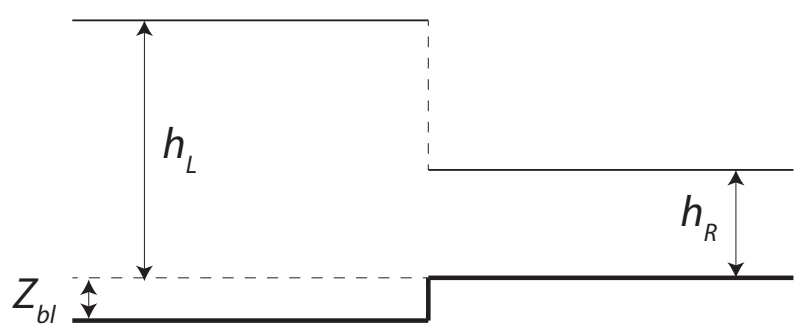

Fig. 2. Scheme of the parameters used in the validation test.

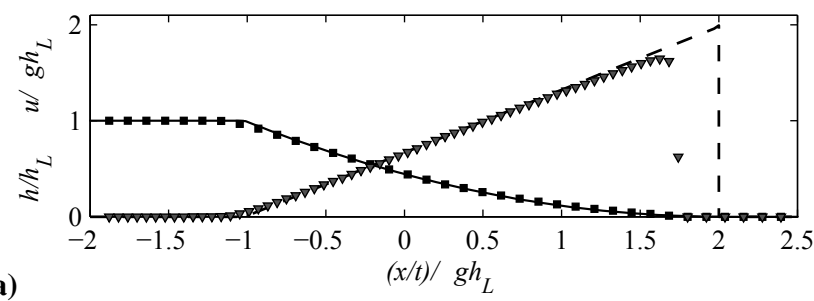

(a)

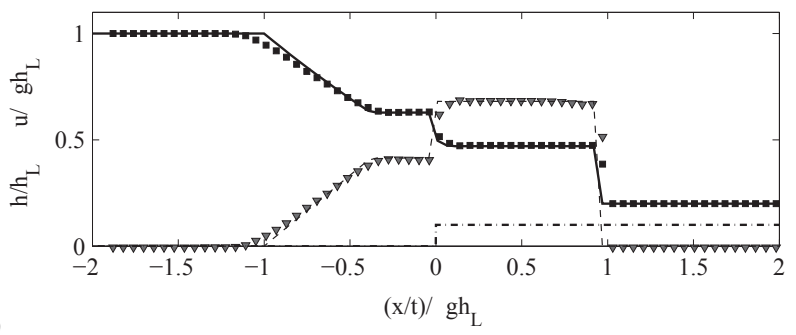

(b)

Fig. 3. Stoker solution with corresponding numerical solution. (a) $h_{\mathrm{L}}=10.0 \mathrm{~m}, h_{\mathrm{R}}=1 \mathrm{~m}, \alpha=0.0$ and $\delta=0.0$; (b) $h_{\mathrm{L}}=10.0 \mathrm{~m}$, $h_{\mathrm{R}}=1 \mathrm{~m}, \alpha=0.18$ and $\delta=-0.09$. Exact solution: $h(-)$, $u$ (- - ); numerical solution with normalised edge length of 0:065: $h(\mathbf{\square}), u(\mathbf{\Delta}), Z_{\mathrm{b}}(-\cdot-)$.

higher depth of the advancing front. This is associated with a decrease in the velocity at the wetting front, which is caused by positivity conditions implemented in the wetting-drying algorithm. When bottom friction is enabled this effect becomes negligible.

Using $h_{\mathrm{L}}=10.0 \mathrm{~m}, \alpha=0.18$ and $\delta=-0.09$, Fig. 3b, the solution features a downstream-progressing expansion wave, an upstream-progressing rarefaction wave and a discontinuity at the bed jump. Again, the numerical results correctly follow the theoretical solution.

These validation tests showed that discontinuous solutions are correctly found by STAV-2D, even though the effects of numerical diffusion are perceptible, particularly in amplitude attenuation at the interface between the left-state and the expansion wave. However, this effect was induced by the coarse grid used in this particular example and becomes meaningless for fine grids. 


\subsection{Laboratory benchmark test}

Laboratory results (Soares-Frazao et al., 2012), were used to validate the model in a dam-break test over mobile bed. The experimental facilities consist of a $3.6 \mathrm{~m}$ wide and $36 \mathrm{~m}$ long flume fully instrumented with flow and bed elevation ultrasonic probes and flow visualisation. The dam is represented by two impervious blocks and a $1.0 \mathrm{~m}$ wide gate, located between the blocks. The bed was a $85 \mathrm{~mm}$ thick layer of sand with a medium diameter of $1.61 \mathrm{~mm}$ and $s=2.56$ (full details can be found in Soares-Frazao et al., 2012). The test employed for validation featured $h_{\mathrm{L}}=0.47 \mathrm{~m}$ and $h_{\mathrm{R}}=0.0 \mathrm{~m}$. The available data consists of measurements of the time-evolution of the water elevation at 8 gauging points and longitudinal final bed profiles, measured in a continuous way from $x=0.5 \mathrm{~m}$ to $x=8 \mathrm{~m}$ with a $\Delta y$ spacing of $0.05 \mathrm{~m}$ (Canelas et al., 2013).

Figures $4 \mathrm{a}$ and $4 \mathrm{~b}$ show the final bed topography and the results obtained with a numerical simulation employing the transport capacity formula from Ferreira et al. (2009), respectively, along with one longitudinal profile (Fig. 4c). The main features of the observed bed profiles are captured by the numerical solutions: strong erosion near the gate and an aggradation wave spanning 3 to $6 \mathrm{~m}$ downstream from the gate. The numerical solution features symmetrical recirculation cells in the space between the gate and the lateral walls (roughly between $x=0.5 \mathrm{~m}$ and $x=2 \mathrm{~m}$ and $|y|>0.5 \mathrm{~m}$, Fig. 4b). The main disagreement between observed and computed bed morphology concerns the pattern of deposition associated with these recirculation cells. In these lateral flow regions, the orientation of the sediment movement is not that of the fluid flow due to secondary currents. Present knowledge on these issues is very limited and was not included in the present model.

This benchmark demonstrated that the model is robust and can simulate discontinuous flows over mobile beds. Its main shortcomings reflect the general insufficiencies of the current state-of-the-art on phenomenological issues related to the interaction between flow and bed morphology. The most relevant issue concerns the parametrization of the effects of secondary currents responsible for the misalignment of sediment transport with the mean velocity vector, a problem common to most models applicable to flows over mobile beds (SoaresFrazao et al., 2012). The sediment transport parameters, presented in Sect. 2.3, were calibrated during this laboratory benchmark and the obtained values were kept unchanged for all the simulations presented in the following sections.

\subsection{Comparison with historical data}

Sedimentological evidence and palaeoecological interpretation studies at Alfeite, on the south bank of the Tagus River, suggest that this area evolved as a low-energy sedimentation environment. Major discontinuities observed in soil properties were most likely caused by tsunamis (Andrade et al.,

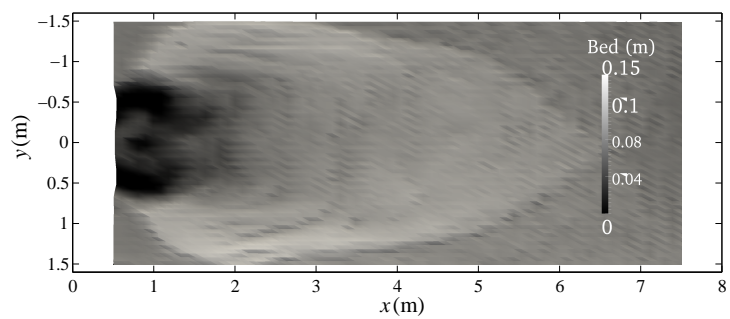

(a) Experimental measurements.

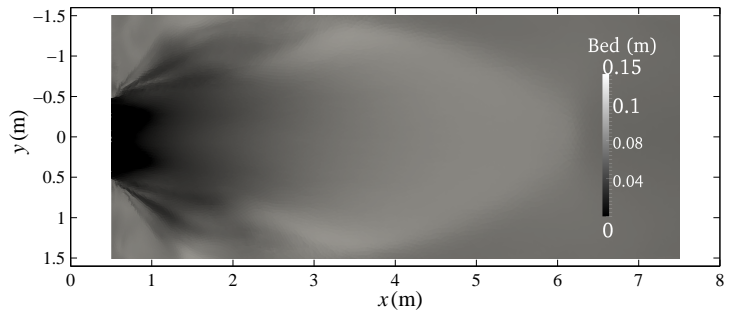

(b) Results for the capacity formula from Ferreira et al. (2009)

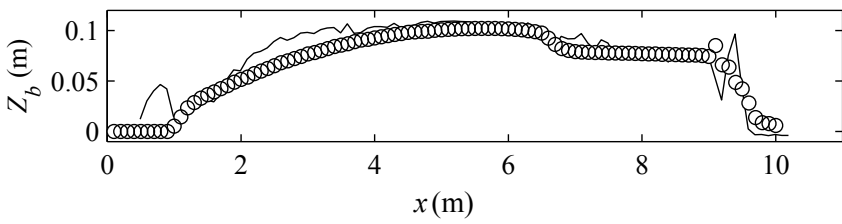

(c) Measured (o) and computed bed elevation (-).
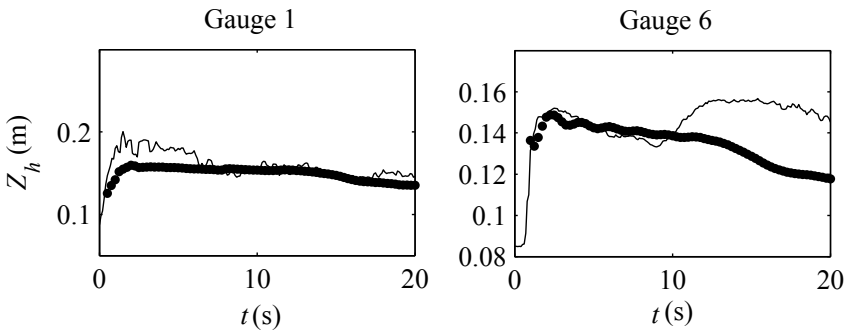

(d) Measured (•) and simulated water (-) level at gauge 1 $(x=0.64 ; y=0.50 \mathrm{~m})$ and gauge $6(x=1.94 ; y=0.33 \mathrm{~m})$.

Fig. 4. Numerical and experimental results from the laboratory benchmark test.

2003) since other high-energy events, such as storm surges, are not capable of carrying sand-size sediment over large distances. Against a background of an average sedimentation rate of 6-8 mmyear $^{-1}$, the high values of both bulk magnetic susceptibility (BMS), nannoplankton concentration and diversity profiles (Fig. 5) are compatible with the AD 1755 and AD 1531 tsunamis (Andrade et al., 2003). These events have severely struck the Tagus estuary and are thought to have transported coastal sediment into the estuary, in accordance with the general principles referred to in Shi and Smith (2003). 

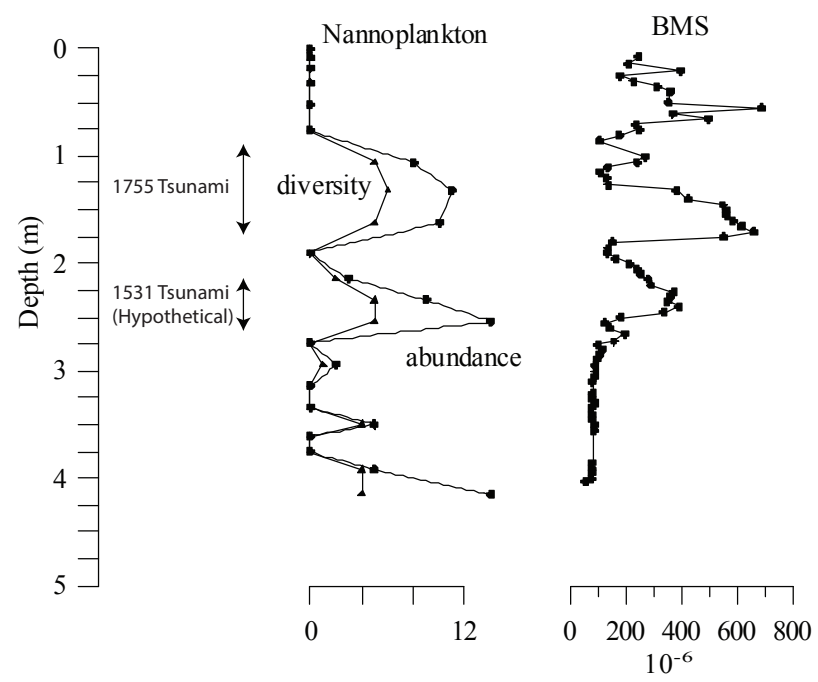

Fig. 5. Profiles of nannoplankton diversity ( $\mathbf{\Delta})$ and concentration (ם) and bulk magnetic susceptibility (BMS) at Alfeite (adapted from Andrade et al., 2003).

The paleo-data summarized in Fig. 5 was employed to validate STAV-2D in what concerns its ability to model sediment transport and bed morphogenesis in complex geometrical conditions. The validation procedure involved simulating a tsunami scenario hydrodynamically similar to that of 1755 , quantifying the sediment deposition layer associated to such event and comparing it to the empirical evidence. Evidently, for this particular test, the model is considered to perform adequately if it features considerable deposition at Alfeite. Specifically the deposition layer must have an order of magnitude compatible with that shown in the statigraphycal records shown in Fig. 5.

The results of bed variation, sediment concentration, water level and velocity obtained with STAV-2D are displayed in Fig. 6. This data concerns 4 computational cells covering the location sampled by Andrade et al. (2003). Figure 6b and $\mathrm{c}$ show that the incoming waves at Alfeite carry high sediment concentrations within the flow column. When velocities drop (Fig. 6d), the particles settle and form a sedimentation layer. The computed sediment layer shown in Fig. 6a is nearly $1.2 \mathrm{~m}$ thick, which is consistent with the profiles shown in Fig. 5, where the high values of Nannoplankton and BMS profiles associated to the 1755 tsunami are represented by a deposit of approximately $0.8-1.2 \mathrm{~m}$.

This test showed that STAV-2D results were consistent with the geological evidence from the 1755 tsunami. Further comparisons of STAV-2D results with historical records, employing the same model parameters, can be found in Conde (2012).

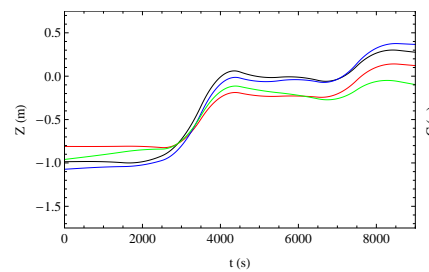

(a) Bed evolution.

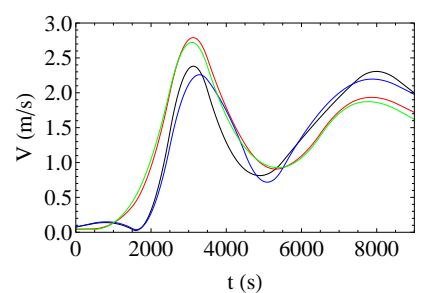

(c) Water level.

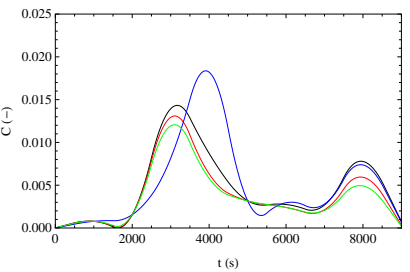

(b) Sediment concentration.

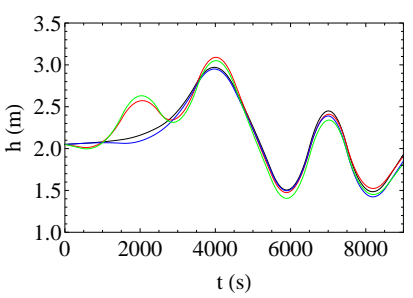

(d) Velocity magnitude.
Fig. 6. STAV-2D results. The colours denote 4 cells that cover the location sampled by Andrade et al. (2003).
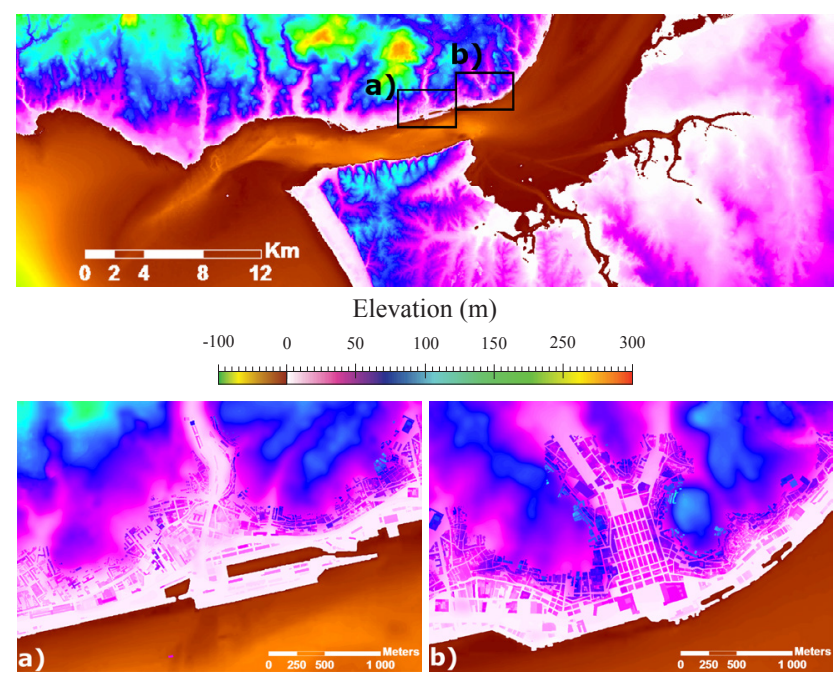

Fig. 7. Digital Elevation Model. Top: overview of the Tagus estuary; bottom: detail of the built environments at Alcântara (a) and downtown Lisbon (b).

\section{Application: a 1755 tsunami in today's Tagus estuary}

\subsection{Initial and boundary conditions}

The well-known tsunami of 1 November 1755 was modelled using STAV-2D employing the current topography of the Tagus estuary. The digital elevation model (DEM) (Fig. 7), including Tagus bathymetry with a $10 \mathrm{~m}$ resolution and $1 \mathrm{~m}$ precision was obtained from Luis (2007). Vectorial data, comprising built environment features, was applied in order to accurately sharpen the definition of the built environment. The resulting DEM is shown in Fig. 7 (bottom). 


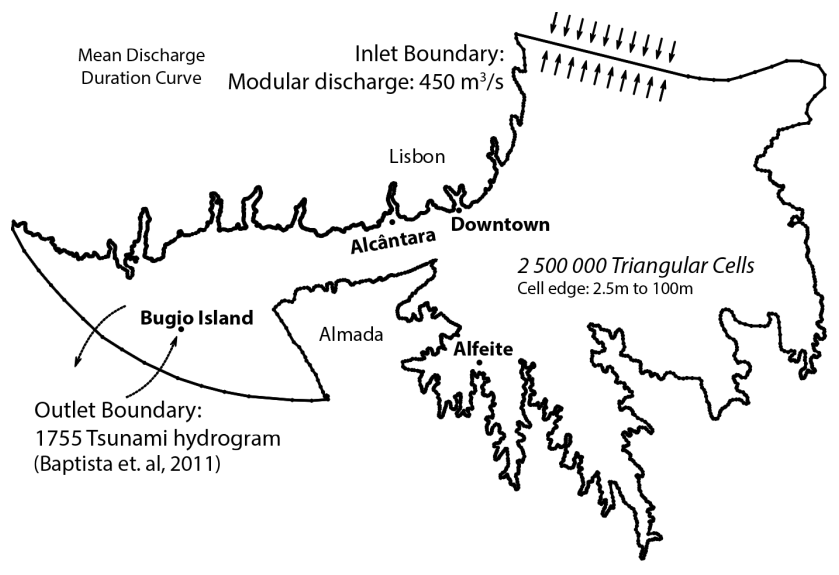

Fig. 8. Simulation setup concerning inner domain, boundaries and relevant locations along the Tagus estuary.

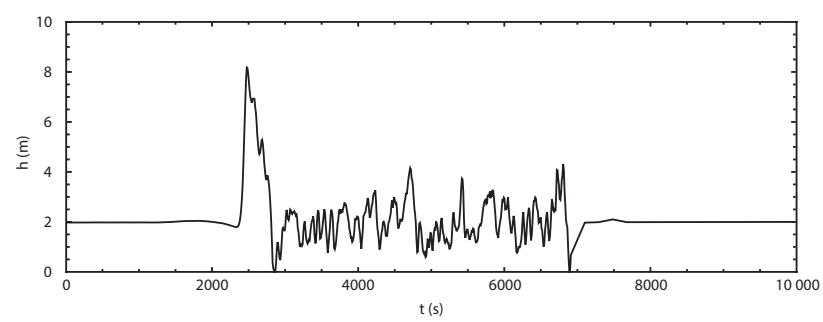

Fig. 9. 1755 Tsunami time series imposed at the Atlantic boundary, provided by Baptista et al. (2011).

Boundary conditions, supported by the Rankine-Hugoniot relations (Conde, 2012), are imposed at the Tagus upstream section and at the Atlantic reach (Fig. 8). In the Tagus Valley inlet, a constant modular discharge of $450 \mathrm{~m}^{3} \mathrm{~s}^{-1}$ flows inside the domain, in a direction normal to that boundary.

Each cell of the Atlantic outlet is forced to follow the 1755 tsunami level time series (Fig. 9), as calculated by Baptista et al. (2011). Historical evidence is one of the most relevant feature of these series, since a wave amplitude of around $5 \mathrm{~m}$ at Bugio is to be expected (Baptista et al., 2011). As the tsunami impacts Bugio Island, STAV-2D prescribed a local water level of about $7.2 \mathrm{~m}$ above hydrographic zero, i.e. $5.2 \mathrm{~m}$ above high tide level.

Initial conditions regarding flow depths and velocities inside the estuary are consistent with a steady flow for a modular discharge and a high tide level of about $2 \mathrm{~mm}$ above hydrographic zero at the Atlantic outlet. The employed unstructured grid was composed of 2500000 triangular cells (Fig. 8), the smallest of which having $2.5 \mathrm{~m}$ edges. The simulation period starts at the instant that the crest of the first wave passes Bugio Island and lasts for $2.7 \mathrm{~h}$, for which the required CPU time was roughly $96 \mathrm{~h}$, using an Intel $i 5$ dualcore $3.2 \mathrm{GHz}$ machine. As for the dam-break examples presented in Sect. 4 the required CPU time was much lower than real time.

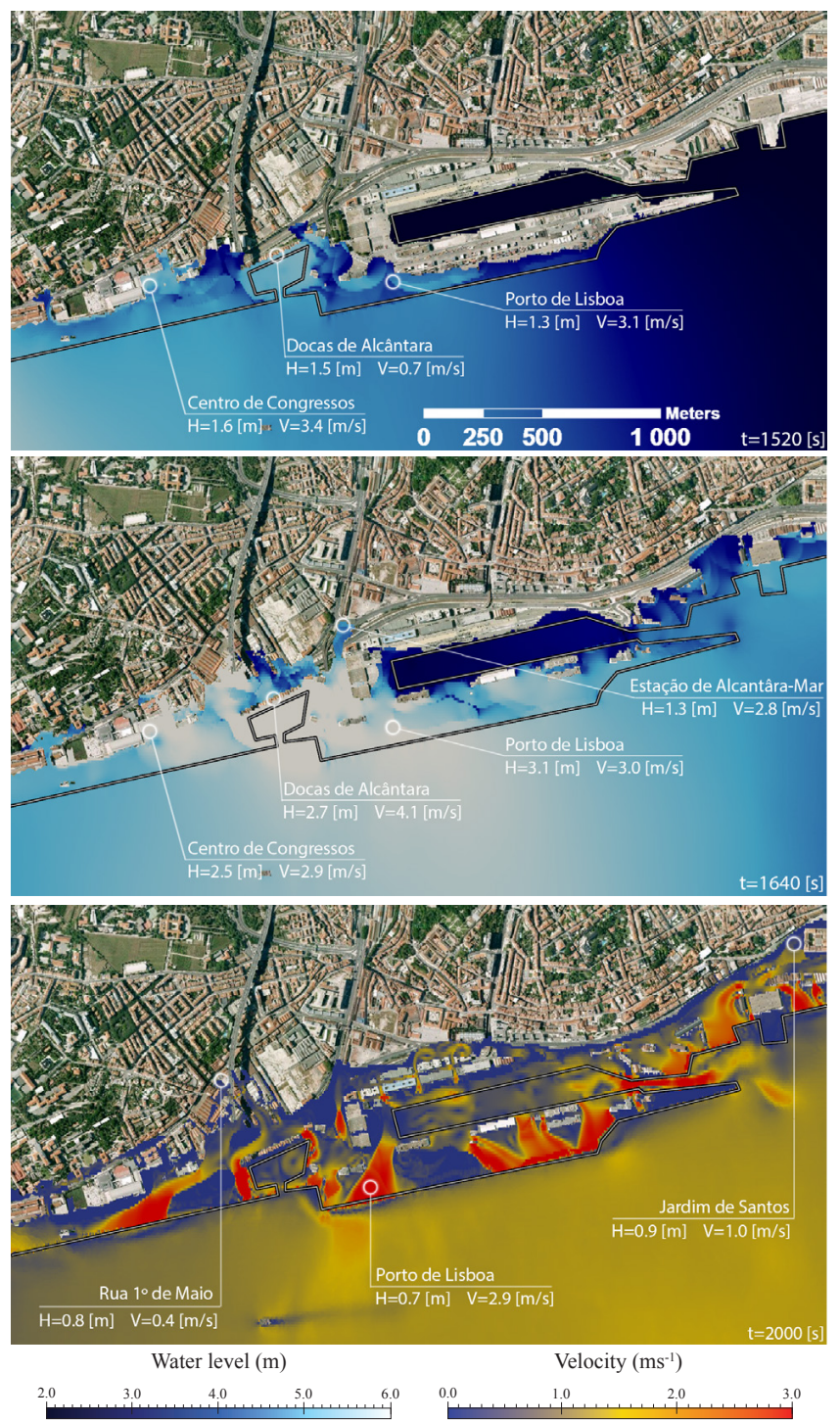

Fig. 10. Results for Alcântara. Water level at $12 \mathrm{~min}$ (top) and 14 min (center); velocities at 20 min (draw-down, bottom).

\subsection{Simulation results}

\subsubsection{Alcântara}

Figure 10 shows the results of a high-tide simulation at Alcântara, 12-20 min after the tsunami hit Bugio, unveiling a 1755-like tsunami that would have had a devastating impact on this riverfront area. Extensively claimed from the Tagus, Alcântara is one of the most worrisome locations in what concerns inundation depths and extent. Inland propagation can travel as far as $550 \mathrm{~m}$, with water depths easily reaching $3 \mathrm{~m}$ at Docas de Alcântara, where velocities reach $4 \mathrm{~ms}^{-1}$. The interaction between buildings and the incoming flow is quite evident, particularly at the harbour, with visible 


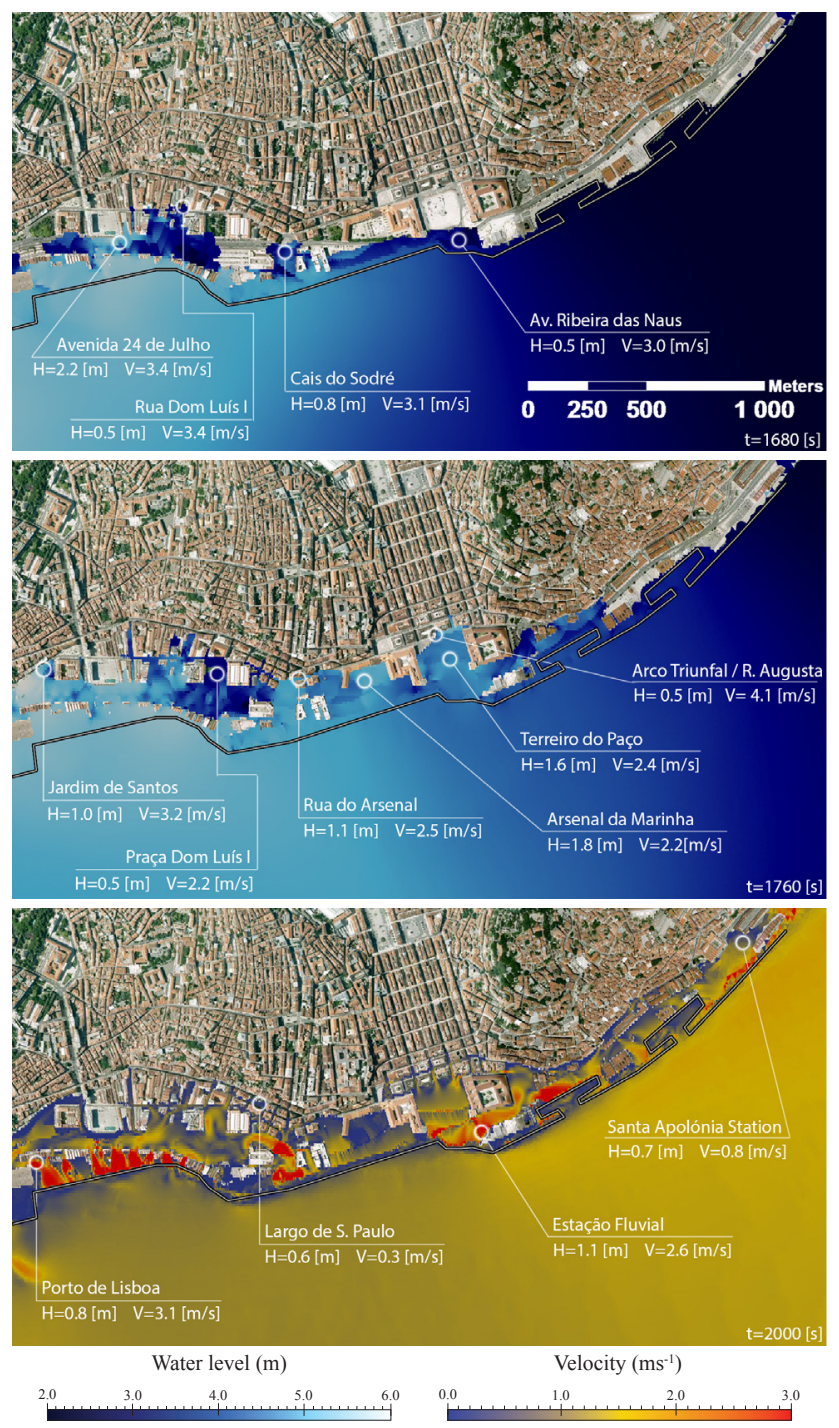

Fig. 11. Results for downtown Lisbon. Water level at $15 \mathrm{~min}$ (top) and $16 \mathrm{~min}$ (center); velocities at $20 \mathrm{~min}$ (draw-down, bottom).

hydrodynamic shadows, wave diffraction and reflection. The inundation only flows through the streets, as seen in the drawdown velocity map (Fig. 10).

\subsubsection{Downtown Lisbon}

Figure 11 shows the results of the same simulation at downtown Lisbon, also evidencing a major impact on this location. Terreiro do Paço would be completely inundated in under one minute as the wave propagates inside the downtown Lisbon orthogonal street meshwork at nearly $4 \mathrm{~ms}^{-1}$. Penetration would reach about $300 \mathrm{~m}$ at downtown and $400 \mathrm{~m}$ at São Paulo. Flow depths were around $1.5 \mathrm{~m}$ at the waterfront, which combined with velocities above $1 \mathrm{~ms}^{-1}$ would be worrisome for unaware pedestrians. Santa Apolonia train station would also be struck by the incoming wave. During

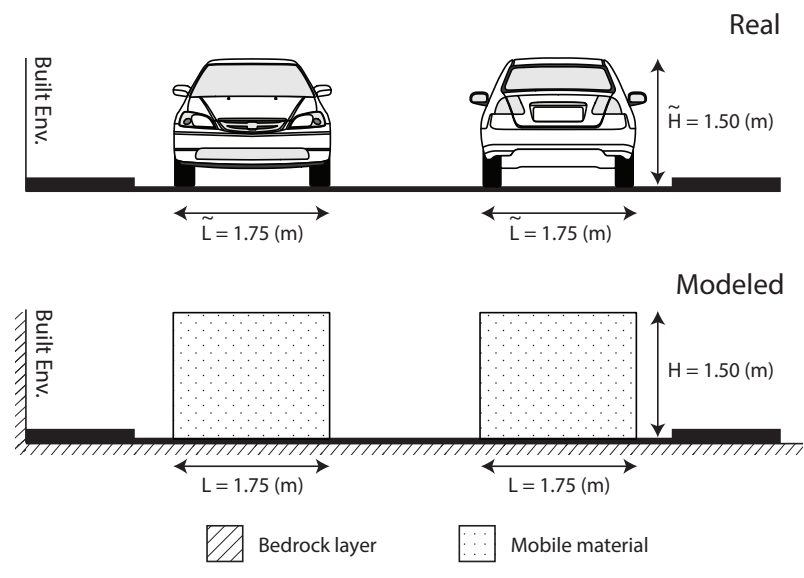

(a) Scheme of the mobile debris setup

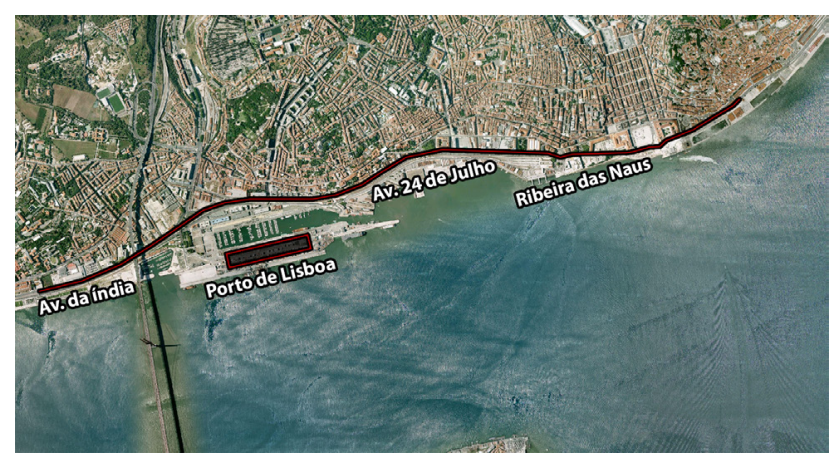

(b) Initial spatial distribution (dark lines and areas)

Fig. 12. Mobile debris setup along Lisbon's riverfront.

draw-down, velocities of 3-4 ms ${ }^{-1}$ would be felt at Terreiro do Paço and Cais do Sodre as the water recedes back to the Tagus through the gaps between riverfront infrastructures.

\subsubsection{Urban debris deposition patterns}

A simplified setup was used to simulate debris transport at Alcântara and downtown Lisbon. A debris specific formulation (Ferreira et al., 2009) was employed when modelling solid transport and a layer of mobile material was added to the base DEM, which was held immobile during this simulation. This layer was composed of vehicle-like volumes (Fig. 12) laid on the main riverfront avenues, with a specified density of $1.5 \mathrm{~kg} \mathrm{~m}^{-3}$ corresponding to that of an average car partially filled with water. The containers at the harbour were modelled with a very similar method.

The aftermath of this scenario showed relevant debris deposition at both designated areas (Fig. 13). At Alcântara, major avenues can become blocked due to debris scattering and accumulation near the riverfront. The downtown area is plentiful in what concerns squares and open spaces, which are specially prone to debris accumulation and may prevent access after the draw-down. 

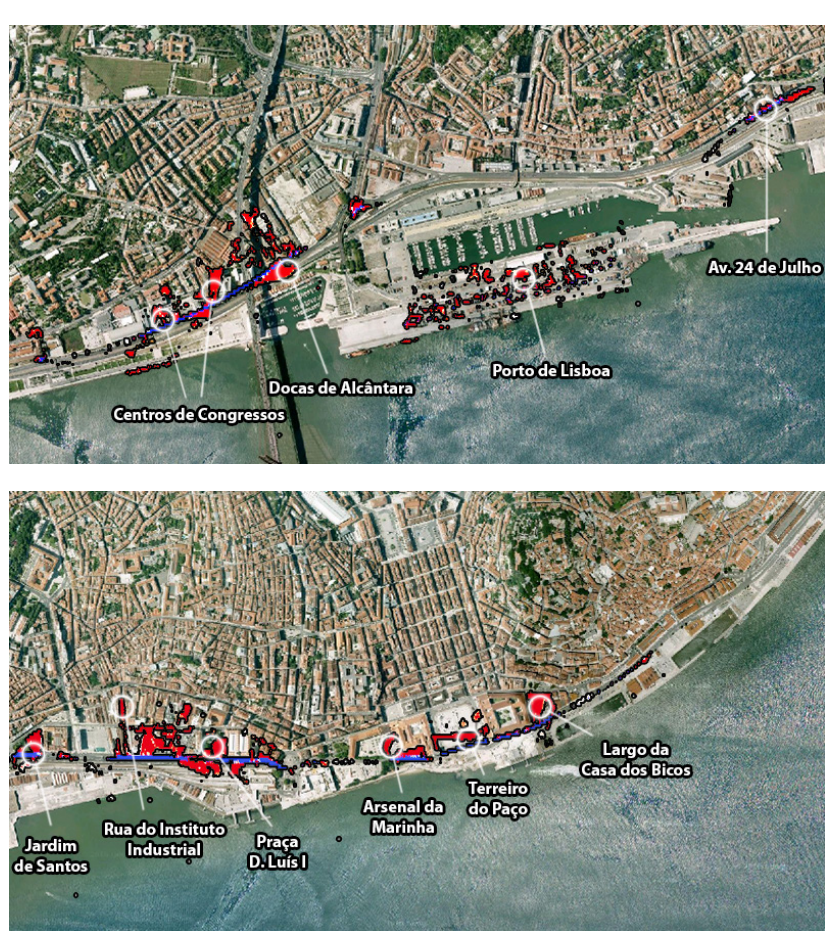

Fig. 13. Final deposition (red) and erosion (blue) patterns at Alcântara (top) and downtown Lisbon (bottom).

\section{Conclusions}

The simulation tool described in this paper, STAV-2D, was shown to be applicable to overland tsunami modelling, since, when propagating inland, tsunamis are shallow flows that carry sediment load, interact with the bottom and may develop bores or other type of discontinuities. The model runs on unstructured grids with representative cell lengths that span from hundreds of metres to $2.5 \mathrm{~m}$. It can thus simultaneously model estuary conditions and the detail of urban meshes at street scale, thus avoiding model nesting.

The model has been developed for applications that involve mobile boundaries, a feature often disregarded in tsunami modelling efforts. It has been validated with theoretical solutions, laboratory measurements and a comparison with paleo-data from the 1755 tsunami.

A forecast exercise was proposed in the paper: a tsunami scenario hydrodynamically similar that of 1755 , modelled for the currently existent bathymetric and altimetric conditions of the Tagus estuary.

The Alcântara-downtown Lisbon riverfront was thoroughly discretized, providing street-level detail in the obtained results. The superposition of a 1755 tsunami and a high-tide initial condition attained severe destruction in this iconic area of Lisbon. Inundation extents spread as far as $300 \mathrm{~m}$ inland in the downtown area and $550 \mathrm{~m}$ at Alcântara. The overland propagating wave would exhibit flow depths ranging from $1 \mathrm{~m}$ to $2 \mathrm{~m}$ along this riverfront. High veloci- ties, mostly peaking at $4-5 \mathrm{~ms}^{-1}$, would be felt at Praça do Comércio and Alcântara during both run-up and draw-down stages. Velocities above $1.5 \mathrm{~ms}^{-1}$ are especially worrisome if combined with water depths higher than waist level, as this often leads to high loss of human lives. Also, high momentum flows incorporate debris (coastal boulders or remains of buildings) into the flow column, increasing its destructive potential.

The morphology and sediment transport features of STAV$2 \mathrm{D}$ also enabled an estimation of the pattern of debris deposition along the riverfront. The computed results suggested major difficulties regarding the post draw-down access in several locations along the Alcântara-downtown Lisbon riverfront.

Overall, this study showed that, regardless of the present day higher ground elevation of the Lisbon riverfront, the city is still dangerously exposed to a tsunami impact. This detailed description of a tsunami impact is expected to support the development of future response mechanisms.

Acknowledgements. Project PTDC/ECM/117660/2010, funded by the Portuguese Foundation for Science and Technology (FCT), has partially supported this work.

Edited by: H. Hebert

Reviewed by: J. Roger and one anonymous referee

\section{References}

Andrade, C., Freitas, M. C., Miranda, J. M., and Baptista, M. A.: Recognizing Possible Tsunami Sediments In The Ultradissipative Environment Of The Tagus Estuary, in: Proceedings of the International Conference on Coastal Sediments 2003, Florida, USA, 18-23, 2003.

Bagnold, R. A.: An approach to the sediment transport problem from general physics, United States Department of Interior, Geological Survey, Professional Paper 442-I, 1966.

Baptista, M. A. and Miranda, J. M.: Revision of the Portuguese catalog of tsunamis, Nat. Hazards Earth Syst. Sci., 9, 25-42, doi:10.5194/nhess-9-25-2009, 2009.

Baptista, M. A., Miranda, J. M., Omira, R., and Antunes, C.: Potential inundation of Lisbon downtown by a 1755-like tsunami, Nat. Hazards Earth Syst. Sci., 11, 3319-3326, doi:10.5194/nhess-113319-2011, 2011.

Canelas, R., Murillo, J., and Ferreira, R. M. L.: Twodimensional depth-averaged modelling of dam-break flows over mobile beds, J. Hydraul. Res., online first, doi:10.1080/00221686.2013.798891, 2013.

Canelas, R. J. F.: 2D Mathematical Modeling of Discontinuous Shallow Sediment-laden Flows, Master's thesis, Instituto Superior Tecnico - Technical University of Lisbon, Portugal, 2010.

Chapman, S. and Cowling, T. G.: The Mathematical Theory of NonUniform Gases, 3rd Edn., Cambridge University Press, Cambridge, UK, 1970. 
Conde, D. A. S.: A tsunami in Lisbon. Where to run?, Master's thesis, Instituto Superior Tecnico - TU Lisbon, Portugal, 2012.

Ferreira, R. M., Franca, M. J., Leal, J. G., and Cardoso, A. H.: Mathematical modelling of shallow flows: Closure models drawn from grain-scale mechanics of sediment transport and flow hydrodynamics, Can. J. Civil. Eng., 36, 1604-1621, 2009.

Ferreira, R. M. L., Leal, J. G., and Cardoso, A. H.: Conceptual model for the bedload layer of gravel bed stream based on laboratory observations, in: River Flow, Balkema, Taylor and Francis Group, 1, 947-956, 2006.

Jenkins, J. T. and Richman, M. W.: Plane simple shear of smooth inelastic circular disks: the anisotropy of the second moment in the dilute and dense limits, J. Fluid Mech., 192, 313-328, 1988.

LeVeque, R.: Finite Volume Methods for Hyperbolic Problems, Cambridge University Press, 2002.

Luis, J. F.: Mirone: A multi-purpose tool for exploring grid data, Comput. Geosci., 33, 31-41, 2007.

Meyer-Peter, E. and Muller, R.: Formulas for bedload transport, in: Proceedings of the 2nd Meeting of the International Association for Hydraulic Structures Research, Stockholm, Sweden, Appendix 2, 39-64, 1948.
Murillo, J. and Garcia-Navarro, P.: Weak solutions for partial differential equations with source terms: application to the shallow water equations, J. Comput. Phys., 229, 4327-4368, doi:10.1016/j.jcp.2010.02.016, 2010.

Pope, S. B.: Turbulent Flows, Cambridge University Press, Cambridge, 2000.

Shi, S. and Smith, D. E.: Coastal Tsunami Geomorphological Impacts And Sedimentation Processes: Case Studies Of Modern And Prehistorical Events, in: Int. Conf. on Estuaries and Coasts, Hangzhou, China, 189-198, 2003.

Soares-Frazao, S. and IAHR Working Group for Dam-break flows: Dam-break flows over mobile beds: experiments and benchmark tests for numerical models, J. Hydraulic Res., 50, 364-375, doi:10.1080/00221686.2012.689682, 2012.

Stoker, J.: Water Waves: The mathematical theory with applications, Interscience Publishers, New York, USA, 1957.

Toro, E.: Shock-Capturing Methods for Free Surface Shallow Flows, Wiley, New York, USA, 2001.

Vazquez-Cendon, M. E.: Improved treatment of source terms in upwind schemes for the shallow water equations with irregular geometry, J. Comput. Phys., 148, 497-526, doi:10.1006/jcph.1998.6127, 1999. 